\title{
Isoscalar mesons and exotic states in light front holographic QCD
}

\author{
Liping Zou, ${ }^{1, *}$ Hans Günter Dosch, ${ }^{1,2, \dagger}$ Guy F. de Téramond, ${ }^{3, *}$ and Stanley J. Brodsky ${ }^{4, \S}$ \\ ${ }^{1}$ Institute of Modern Physics, Chinese Academy of Sciences, Lanzhou 730000, China \\ ${ }^{2}$ Institut für Theoretische Physik der Universität, D-69120 Heidelberg, Germany \\ ${ }^{3}$ Laboratorio de Física Teórica y Computacional, Universidad de Costa Rica, 11501 San José, Costa Rica \\ ${ }^{4}$ SLAC National Accelerator Laboratory, Stanford University, Stanford, California 94309, USA
}

(Received 7 February 2019; published 26 June 2019)

\begin{abstract}
In this article a systematic quantitative analysis of the isoscalar bosonic states is performed in the framework of supersymmetric light front holographic QCD. It is shown that the spectroscopy of the $\eta$ and $h$ mesons can be well described if one additional mass parameter-which corresponds to the hard breaking of chiral $U(1)$ symmetry in standard QCD—is introduced. The mass difference of the $\eta$ and $\eta^{\prime}$ isoscalar mesons is then determined by the strange quark mass content of the $\eta^{\prime}$. The theory also predicts the existence of isoscalar tetraquarks which are bound states of diquarks and antidiquarks. The candidates for these exotic isoscalar tetraquarks are identified. In particular, the $f_{0}(1500)$ is identified as isoscalar tetraquark; the predicted mass value $1.52 \mathrm{GeV}$ agrees with the measured experimental value within the model uncertainties.
\end{abstract}

DOI: 10.1103/PhysRevD.99.114024

\section{INTRODUCTION}

In a series of recent papers [1-7] it was shown that lightfront holographic QCD (LFHQCD) [8-10], especially after implementation of superconformal algebra [1,2] (supersymmetric LFHQCD), explains many spectroscopic and dynamical features of the observed hadrons, thus providing nontrivial analytic connections between the spectroscopy of hadrons and the dynamics underlying observables such as their form factors and quark distributions [11,12]. The LFHQCD formalism is based on Lorentz-invariant bound-state light-front Schrödinger equations with a single mass parameter appearing in a color-confining potential. Mesons, baryons, and tetraquarks are unified as components of a supersymmetric multiplet.

LFHQCD is inspired by the Maldacena conjecture [13]: a weakly coupled classical five-dimensional gravitational theory with anti-de Sitter (AdS) metric is a holographical dual to a strongly coupled four-dimensional quantum gauge field theory defined at the spacetime boundary of $\mathrm{AdS}_{5}$. This resulting four-dimensional field theory is a superconformal gauge theory in the limit of $N_{C} \rightarrow \infty$ colors.

\footnotetext{
*zoulp@impcas.ac.cn

†h.g.dosch@thphys.uni-heidelberg.de

${ }^{*}$ gdt@asterix.crnet.cr

sjbth@slac.stanford.edu
}

Published by the American Physical Society under the terms of the Creative Commons Attribution 4.0 International license. Further distribution of this work must maintain attribution to the author(s) and the published article's title, journal citation, and DOI. Funded by SCOAP ${ }^{3}$.
A crucial feature of LFHQCD is the correspondence between the fields of the five-dimensional theory and those of the four-dimensional theory at fixed light front time $\tau=x^{+}=x^{0}+x^{3}$. This remarkable correspondence is based on the observation that the classical equations of motion derived from the action of the five-dimensional theory have the identical form as bound-state equations for two massless constituents in light front (LF) quantization. This holographic correspondence is also realized dynamically as the analytic equality of the AdS and light front expressions for electromagnetic and gravitational form factors of the composite states $[14,15]$.

Therefore in [9] light front holography was proposed as the first semiclassical approximation to QCD. The equations of motion, derived from the five-dimensional AdS modified action, are mapped to the wave equations of a system of two massless color-confined constituents in QCD quantized on the light front; the fifth coordinate in $\mathrm{AdS}_{5}$, the holographic variable $z$, is identified with the boostinvariant transverse LF separation $\zeta$ (see Appendix A). In this approach, the "dictionary" between the bound-state wave functions of the classical five-dimensional theory and the four-dimensional theory quantized on the light front is fixed: for example, the bound state consisting of a confined color-singlet quark and an antiquark with orbital and total angular momentum zero represents a pseudoscalar meson.

The implementation of the superconformal algebra in LFHQCD uniquely determines the form of the interaction. It also explains quantitatively the striking similarities between baryon and meson spectra [2-5] and fixes the modification of the AdS action. One predicts universal 
Regge slopes in the radial and orbital quantum numbers $n$ and $L$ for both mesons and baryons. Only one mass parameter appears. A striking success of LFHQCD is the prediction of a massless pion $[9,10]$ in the chiral limit. This is a consequence of the implementation of the superconformal algebra, in contrast to the Goldstone mechanism in a theory with a degenerate vacuum.

Isospin is not introduced explicitly in LFHQCD; therefore massless particles occur not only in the isovector but also in the isoscalar sector-a sector which has proven to be particularly challenging to explain since the explicit breaking of the chiral $U(1)$ symmetry of standard QCD is required. We will show in this article that the spectroscopy of $\eta$ - and $h$-meson states can be quantitatively described by LFHQCD with the introduction of a single-parameter modification of the LF Hamiltonian. A remarkable feature of the present approach is that the numerical value of the additional parameter coincides numerically with the LFHQCD confinement scale $\lambda$; this could point to a deeper connection since in the chiral limit there is only one available scale in LFHQCD, the hadronic mass scale $\lambda$.

A consequence of the introduction of supersymmetry is the occurrence of tetraquarks as the second partners of the baryons $[3,6,16]$. Since extra hadronic states appear to be particularly abundant in the isoscalar channels, it is promising to look specifically for possible tetraquarks in those channels. There is a tremendous amount of literature on tetraquarks, and one can easily be lost in the possibilities. Supersymmetric LFHQCD, however, has the advantage that it makes quantitative and well defined predictions of the masses of these states. We will therefore perform a quantitative analysis of all of the tetraquark candidates among the isoscalar bosons. In this respect the present analysis is complementary to a previous analysis [6] which gave a general overview over all possible tetraquark states predicted by supersymmetric LFHQCD. In this paper we give predictions for the masses of bosonic hadrons which can be specifically identified as tetraquarks within the extended LFHQCD scheme described here.

The paper is organized as follows: In Sec. II we briefly review the main theoretical ingredients of LFHQCD. We then extend the approach to $\eta$ and $h$ mesons and discuss the implications for the Pauli principle on the quantum numbers of tetraquarks. In Sec. III we compare theory with experiment in all bosonic channels and predict the candidates which are most likely isoscalar tetraquarks. The possible relation of this modification of the LF Hamiltonian to chiral $U(1)$ breaking in standard QCD is briefly discussed in the last section, Sec. IV.

\section{PRINCIPAL RESULTS OF LFHQCD AND SUPERSYMMETRIC LFHQCD}

In this section we will review the main theoretical results for the hadron spectroscopy predicted by supersymmetric LFHQCD; a more detailed treatment is given in Refs. $[2,3,10,16]$. Some intermediate steps, which are most important for this paper, are given in Appendix A.

As other holographic "bottom-up" models, LFHQCD starts from an invariant action in a five-dimensional space with the metric of $\mathrm{AdS}_{5}$. Due to the maximal symmetry of the $\mathrm{AdS}_{5}$ action, the corresponding four-dimensional theory is invariant under the conformal group. This symmetry has to be broken by introducing a mass scale. After such a modification, the resulting classical equations of motion of the five-dimensional theory have the form of Hamiltonian equations for bound states of two massless quarks, where the $q \bar{q}$ interaction is determined by the assumed modification of the invariant $\mathrm{AdS}_{5}$ action. The unique form of the modified $\mathrm{AdS}_{5}$ action can in fact be completely determined by a symmetry principle: the resulting Hamiltonian of our semiclassical theory must be contained within the superconformal algebra. ${ }^{1}$ This requirement completely determines the form of the color-confining $q \bar{q}$ interaction and consequently the modification of the $\mathrm{AdS}_{5}$ action, for both mesons and baryons [1,2]. It also explains the observed approximate degeneracy between baryon and meson spectra and predicts the masses of the tetraquark states [3].

The resulting hadronic spectrum has the form of a supersymmetric 4-plet of $q \bar{q}$ mesons $(M)$, quark + diquark baryons $(B)$, and diquark + antidiquark tetraquarks $(T)$, where in $S U(3)_{C}$ the diquark cluster has color $\overline{3}_{C}$. The predicted hadron masses can, in the limit of massless quarks, be summarized in the following formulas [3]:

$$
\begin{gathered}
M_{M}^{2}=4 \lambda\left(n+L_{M}\right)+2 \lambda \mathcal{S}, \\
M_{B}^{2}=4 \lambda\left(n+L_{B}+1\right)+2 \lambda \mathcal{S}, \\
M_{T}^{2}=4 \lambda\left(n+L_{T}+1\right)+2 \lambda \mathcal{S} .
\end{gathered}
$$

Here $n$ denotes the radial excitation quantum number, $L_{M}$ denotes the LF orbital angular momentum between the quark and antiquark in the meson, $L_{B}$ denotes that between the diquark cluster and the quark in the baryon, and $L_{T}$ denotes that between the two diquark clusters in the tetraquark. $\mathcal{S}$ is for mesons the total quark spin, and for baryons and tetraquarks the minimal possible quark spin of a diquark cluster inside the hadron. In supersymmetric LFHQCD only mesons with $J=L+\mathcal{S}, \mathcal{S}=0$ or 1 , can be considered. The scale $\lambda$ is the only free constant of supersymmetric LFHQCD in the limit of massless quarks.

As mentioned above, the multiplets of supersymmetric LFHQCD contain only mesons, baryons and tetraquarks with total spin of the hadron $J=L+\mathcal{S}, \mathcal{S}=0$ or 1 . But once the modification of the $\mathrm{AdS}_{5}$ action is fixed by the superconformal constraints, one can apply this

\footnotetext{
${ }^{1}$ The quantum field theory underlying the Maldacena conjecture is a superconformal theory.
} 
TABLE I. Mass corrections according to (4) in $\mathrm{GeV}^{2}$.

\begin{tabular}{ll}
\hline \hline & $\Delta M^{2}\left[m_{q}, m_{q}\right]=(0.14)^{2}$ \\
& $\Delta M^{2}\left[m_{s}, m_{s}\right]=(0.773)^{2}$ \\
& $\Delta M^{2}\left[m_{q}, m_{q}, m_{q}, m_{q}\right]=(0.344)^{2}$ \\
& $\Delta M^{2}\left[m_{q}, m_{s}, m_{q}, m_{s}\right]=(0.959)^{2}$ \\
& $\Delta M^{2}\left[m_{s}, m_{s}, m_{s}, m_{s}\right]=(1.53)^{2}$ \\
\hline \hline
\end{tabular}

modification also in normal LFHQCD and derive the Hamiltonian for mesons with quark spin $\mathcal{S}=1$ and $J=$ $L$ or $J=L-1$. In this way we can also compare theory with the observed mesons with $J^{P C}=0^{++}$and $1^{++}$. In this case the quantity $\mathcal{S}$ in (1) is replaced by $(J-L)$; see [17].

In order to incorporate the effects of quark masses in LF theory, at least at lowest order, one can include the invariant mass term $\sum_{i} \frac{m_{i}^{2}}{x_{i}}$ to the LF Hamiltonian-the contribution of quark masses to the LF kinetic energy. To first approximation, this leaves the confining LF potential unchanged. For a state containing $N$ quarks with masses $m_{1}, \ldots, m_{N}$ one then obtains the quadratic mass shift [3],

$$
\Delta M^{2}\left[m_{1}, \ldots, m_{N}\right]=\lambda^{2} \frac{\partial}{\partial \lambda} \log F
$$

with

$$
F[\lambda]=\int_{0}^{1} d x_{1} \cdots \int_{0}^{1} d x_{N} e^{-\frac{1}{\lambda}\left(\sum_{i=1}^{N} \frac{m_{i}^{2}}{x_{i}}\right)} \delta\left(\sum_{i=1}^{N} x_{i}-1\right)
$$

The quark mass corrections lead to a modified mass spectroscopy for the bosons:

$$
\begin{array}{r}
M_{M}^{2}=4 \lambda\left(n+L_{M}\right)+2 \lambda(J-L)+\Delta M^{2}\left[m_{1}, m_{2}\right], \\
M_{T}^{2}=4 \lambda\left(n+L_{T}+1\right)+2 \lambda \mathcal{S}+\Delta M^{2}\left[m_{1}, m_{2}, m_{3}, m_{4}\right] .
\end{array}
$$

The value of $\lambda$ has been fitted previously [3] to the full hadron spectrum with the result $\sqrt{\lambda}=\kappa=0.523 \pm$ $0.025 \mathrm{GeV}$. The effective masses of the light and strange quarks were determined in [10] from $m_{\pi}^{2}=\Delta M^{2}\left[m_{q}, m_{q}\right]$ and $m_{K}^{2}=\Delta M^{2}\left[m_{s}, m_{q}\right]$, which yields the values $m_{q}=$ 0.046 and $m_{s}=0.357 \mathrm{GeV}$. In Table I the numerical values of the mass corrections for nonstrange and strange quark masses, according to (4), are collected. The numerical results for the boson masses $M_{M}$ and $M_{T}$ according to (6) and (7) are given in Table II.

\section{A. $\boldsymbol{\eta}$ and $\boldsymbol{h}$ mesons}

As can be seen from (1) the ground states $(n=0)$ of pseudoscalar mesons with angular momentum $J=L=0$ in LFHQCD have zero mass in the limit of massless quarks. This prediction of a massless pseudoscalar meson $q \bar{q}$ bound state is a remarkable success of LFHQCD for the
TABLE II. Boson masses according to (6) and (7) in GeV.

\begin{tabular}{llllll}
\hline \hline & \multicolumn{5}{c}{$M[\mathrm{GeV}]$} \\
\cline { 2 - 6 } Quant. num. & \multicolumn{5}{c}{ Quark content } \\
\hline$n+L+\frac{J-L}{2}$ & $\bar{q} q_{0}$ & $\bar{s} s_{0}$ & $\overline{q q} q q$ & $\overline{q s} q s$ & $\overline{s s} s s$ \\
\hline 0 & 0.14 & 0.77 & 1.1 & 1.42 & 1.85 \\
$1 / 2$ & 0.753 & 1.07 & 1.33 & 1.6 & 2.0 \\
1 & 1.06 & 1.3 & 1.52 & 1.76 & 2.13 \\
$3 / 2$ & 1.29 & 1.49 & 1.69 & 1.91 & 2.25 \\
2 & 1.49 & 1.67 & 1.84 & 2.05 & 2.37 \\
$5 / 2$ & 1.66 & 1.82 & 1.99 & 2.18 & 2.48 \\
3 & 1.82 & 1.97 & 2.12 & 2.3 & 2.59 \\
$7 / 2$ & 1.96 & 2.1 & 2.24 & 2.42 & 2.7 \\
4 & 2.1 & 2.23 & 2.36 & 2.53 & 2.79 \\
$9 / 2$ & 2.22 & 2.35 & 2.48 & 2.63 & 2.89 \\
5 & 2.34 & 2.46 & 2.58 & 2.74 & 2.98 \\
\hline \hline
\end{tabular}

isovector channel. But since LFHQCD does not treat flavor explicitly, this result also applies to the isoscalar channel. In this case, however, the least massive observed hadron is the $\eta$ meson, which has a mass of $0.548 \mathrm{GeV}$, much heavier than the pion.

In standard QCD the difference between the isovector and isoscalar sector is generally attributed to a hard breaking of the chiral $U(1)$ symmetry of the classical QCD Lagrangian by nonperturbative effects [18-26]. Since flavor is not treated explicitly in LFHQCD we will treat that breaking phenomenologically by ensuring that the lowest $I=0, J=\mathcal{S}=0$ meson has the correct mass $m_{\eta}=$ $0.548 \mathrm{GeV}$ [27]. This can be achieved by adding to the LF Hamiltonian the constant term $\Delta_{\eta}^{2} \lambda \delta_{\mathcal{S} 0} \delta_{I 0}$, with

$$
\Delta_{\eta}^{2}=\frac{1}{\lambda}\left(M_{\eta}^{2}-\Delta M^{2}\left[m_{q}, m_{q}\right]\right)=\frac{1}{\lambda}\left(M_{\eta}^{2}-M_{\pi}^{2}\right),
$$

a dimensionless quantity with the value $\Delta_{\eta}^{2}=1.03 \pm 0.08$. This additional term encodes a hard chiral $U(1)$ breaking in standard QCD. In the isoscalar vector channel current conservation in QCD forbids an anomaly; accordingly, the effective light front Hamiltonian should not be modified in this sector. The scale associated with the $\eta$ mass, namely $\lambda \Delta_{\eta}^{2}$, numerically nearly coincides with the confinement scale $\lambda=(0.523 \pm 0.025 \mathrm{GeV})^{2}$ [3]. It is therefore tempting to speculate there is a deeper connection behind this numerical equality and that the two scales are indeed the same since there is a unique mass scale in LFHQCD. We will, however, not discuss this issue further in this article.

The resulting mass formulas for mesons and tetraquarks in the isoscalar sector are

$$
\begin{aligned}
M_{M}^{2}= & 4 \lambda\left(n+L_{M}\right)+\Delta M^{2}\left[m_{1}, m_{2}\right]+2 \lambda \mathcal{S}+\lambda \Delta_{\eta}^{2}, \\
M_{T}^{2}= & 4 \lambda\left(n+L_{T}+1\right)+\Delta M^{2}\left[m_{1}, m_{2}, m_{3}, m_{4}\right] \\
& +2 \lambda \mathcal{S}+\lambda \Delta_{\eta}^{2},
\end{aligned}
$$


where $\Delta_{\eta}^{2}$ is given by (8). The satisfactory comparison with experiment will be given in Sec. III.

\section{B. Isospin and spin of tetraquarks}

The two quarks of a diquark cluster in a tetraquark are antisymmetric in color; the spin-statistics theorem therefore demands that they are symmetric in the remaining quantum numbers. Therefore a diquark cluster with specific isospin and relative orbital angular momentum zero must have isospin and spin either both equal to zero or both equal to 1 . This implies that the lowest lying tetraquark with isospin 0 must have total angular momentum $J=0$ and the one with isospin 1 must have $J=1$; as a result, the squared masses of the two states differ by $2 \lambda$.

These arguments do not apply to tetraquarks containing constituents which are not related by isospin symmetry. The lowest state consisting of the type $(\overline{q s} q s)$ is isospin degenerate and has in supersymmetric LFHQCD the mass $1.42 \mathrm{GeV}$. Since diquark clusters are bosons, the parity and $C$-parity of a tetraquark with $\mathcal{S}=0$ is $(-1)^{L}$. For a tetraquark with $\mathcal{S}=1$ both $C$-parities are possible.

For a tetraquark consisting only of strange and antistrange quarks, the spin-statistics theorem requires that the total spin of each diquark cluster is 1 . Therefore such tetraquarks are not predicted in the scheme of supersymmetric LFHQCD. We will therefore restrict our general discussion to tetraquarks, where only one diquark cluster has spin 1. But at least tentatively, we suggest that the higher lying states of $f_{2}$ mesons might contain admixtures of tetraquarks $(\overline{s s} s s)$ and extend (7) to $\mathcal{S}=2$. This is motivated by the appearance of two $\phi$ mesons in the decay channels of these mesons.

\section{COMPARISON WITH EXPERIMENT}

The augmented LFHQCD theory presented here contains four parameters. Three of them, the scale $\sqrt{\lambda}=\kappa=$ $0.523 \mathrm{GeV}$ and the quark masses $m_{q}=0.045, m_{s}=$ $0.357 \mathrm{GeV}$, are taken from previous analyses of the hadron spectrum [3]. For this analysis the shift term $\Delta_{\eta}^{2}=\lambda_{\eta} \delta_{\mathcal{S} 0} \delta_{I 0}$ has been introduced in the LF Hamiltonian for mesons (9) and tetraquarks (10) in the isoscalar sector. As discussed above, the scale $\Delta_{\eta}^{2} \lambda$ is fixed by the $\eta$ mass, $\Delta_{\eta}^{2} \lambda \equiv$ $M_{\eta}^{2}-M_{\pi}^{2} \simeq \lambda$.

\section{A. $\boldsymbol{\eta}$ and $\boldsymbol{h}$ mesons}

As can be seen from Fig. 1, the mass difference $\sqrt{M_{\eta^{\prime}}^{2}-M_{\eta}^{2}}$ agrees reasonably well with the corresponding mass differences between other hadrons $x$ and $y$ with the same external quantum numbers. This quantity is determined by the mass difference between the strange and the light quarks: two light quarks are replaced by two strange quarks in baryons, and for mesons the comparison is made for isoscalar mesons with the same quantum numbers. If the

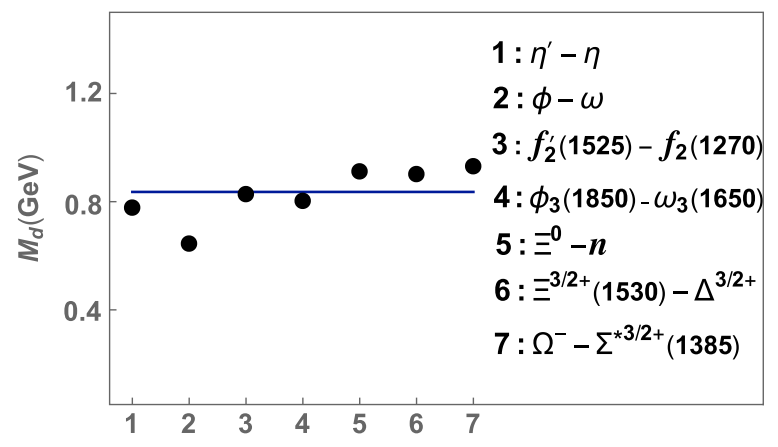

FIG. 1. The mass differences $M_{d}=\sqrt{M_{x}^{2}-M_{y}^{2}}$ between states with the same $J^{P C}$ and $J^{P}$, respectively.

hadrons $x$ and $y$ are mesons, one can conclude from their decays that the heavier meson $x$ is predominantly an $\bar{s}-s$ pair, whereas the lighter hadron $y$ is a $\bar{q}-q$ pair, both with the same quark spin $\mathcal{S}$. If the hadrons $x$ and $y$ are baryons, then they both have the same quantum numbers $J^{P}$, but the strangeness of the heavier one is larger by two units; i.e., the $q-q$ diquark cluster of the lighter baryon $y$ is replaced by an $s-s$ cluster in the heavier baryon $x$. The mean value of the mass difference $M_{d}=\sqrt{M_{x}^{2}-M_{y}^{2}}$ is $0.84 \mathrm{GeV}$ with a standard deviation of $0.09 \mathrm{GeV}$; the theoretically predicted value is $\sqrt{\Delta^{2}\left[m_{s}, m_{s}\right]-\Delta^{2}\left[m_{q}, m_{q}\right]}=0.76 \mathrm{GeV}$ (see Table I).

Therefore in the present analysis the $\eta-\eta^{\prime}$ mass difference, sometimes referred to as the $\eta-\eta^{\prime}$ puzzle, is determined by the strange quark mass contribution

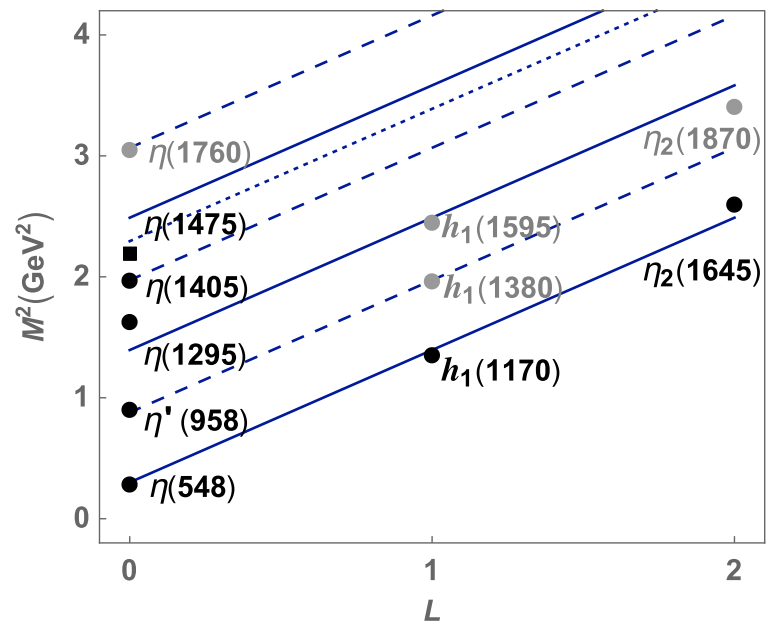

FIG. 2. The $I=0, J=L$, and $C=(-1)^{L}$ states. The theoretical predictions for the meson trajectories are from (9): Continuous lines are for $\eta$ families, and dashed lines are for $\eta^{\prime}$ families. Data (filled circles and square) are from PDG [27], and unconfirmed states are in gray. 
TABLE III. All confirmed light isoscalar bosonic states. The data are from the PDG [27], and the theoretical results from [(6), (7)] and [(9), (10)]. The label $d$ signifies that channels with open or hidden strangeness are dominant; for the $\eta^{\prime}$ decay see the note in the text. If a state with $I=1$, but equal residual quantum numbers occur, it is indicated in the last column. For further explanation see the text.

\begin{tabular}{|c|c|c|c|c|c|c|c|c|}
\hline \multicolumn{4}{|c|}{ Experiment } & \multicolumn{5}{|c|}{ Theory } \\
\hline \multirow[t]{2}{*}{$J^{P C}$} & \multirow[t]{2}{*}{ Name } & \multirow[t]{2}{*}{$M[\mathrm{MeV}]$} & \multirow[t]{2}{*}{ Decay } & \multicolumn{2}{|c|}{ Meson } & \multicolumn{2}{|c|}{ Tetraquark } & \multirow{2}{*}{$I=1$ partner } \\
\hline & & & & $M_{n L, J-L}$ & {$[\mathrm{GeV}]$} & $T_{n L, \mathcal{S}}$ & {$[\mathrm{GeV}]$} & \\
\hline$\overline{0^{-+}}$ & $\eta$ & 548 & & $\bar{q} q_{000}^{\eta}$ & $(0.548)$ & & & \\
\hline $0^{-+}$ & $\eta^{\prime}(958)$ & 958 & & $\bar{s} s_{000}^{\eta}$ & 0.94 & & & \\
\hline $0^{-+}$ & $\eta(1295)$ & $1294 \pm 4$ & & $\bar{q} q_{100}^{\eta}$ & 1.18 & & & \\
\hline $0^{-+}$ & $\eta(1405)$ & $1409 \pm 2$ & & $\bar{s} s_{100}^{\eta}$ & 1.41 & & & \\
\hline $0^{-+}$ & $\eta(1475)$ & $1476 \pm 4$ & $\mathrm{~d}$ & $\bar{s} s_{? 00}^{\eta}$ & & & & \\
\hline $0^{++}$ & $f_{0}(500)$ & $475 \pm 75$ & & & & & & \\
\hline $0^{++}$ & $f_{0}(980)$ & $990 \pm 20$ & & $\bar{q} q_{01-1}$ & 0.75 & $(\overline{q q} q q)_{000}$ & 1.10 & $a_{0}(980)$ \\
\hline $0^{++}$ & $f_{0}(1370)$ & $1350 \pm 6$ & & $\bar{q} q_{11-1}$ & 1.29 & & & \\
\hline $0^{++}$ & $f_{0}(1500)$ & $1504 \pm 6$ & & & & $(\overline{q q} q q)_{100}$ & 1.52 & \\
\hline $0^{++}$ & $f_{0}(1710)$ & $1723 \pm 6$ & d & $\bar{s} s_{21-1}$ & 1.82 & & & \\
\hline $1^{--}$ & $\omega$ & 783 & & $\bar{q} q_{001}$ & 0.75 & & & $\rho$ \\
\hline $1^{--}$ & $\omega(1420)$ & $1425 \pm 25$ & & $\bar{q} q_{101}$ & 1.29 & $(\overline{q q} q q)_{010}$ & 1.52 & $\rho(1450)$ \\
\hline $1^{--}$ & $\omega(1650)$ & $1670 \pm 30$ & & $\bar{q} q_{201}$ & 1.66 & & & \\
\hline $1^{--}$ & $\phi$ & 1019 & d & $\bar{s} s_{001}$ & 1.07 & & & \\
\hline $1^{--}$ & $\phi(1680)$ & $1680 \pm 20$ & d & $\bar{s} s_{101}$ & 1.49 & $(\overline{s q} s q)_{010}$ & 1.76 & \\
\hline $1^{--}$ & $\phi(2170)$ & $2188 \pm 10$ & & $\bar{s} s_{301}$ & 2.10 & & & \\
\hline $1^{+-}$ & $h_{1}(1170)$ & $1170 \pm 20$ & & $\bar{q} q_{010}^{\eta}$ & 1.18 & & & \\
\hline $1^{++}$ & $f_{1}(1285)$ & 1282 & & $\bar{q} q_{110}$ & 1.49 & & & $a_{1}(1260)$ \\
\hline $1^{++}$ & $f_{1}(1420)$ & 1426 & d & $\bar{s} s_{010}$ & 1.30 & $(\overline{s q} s q)_{001}$ & 1.60 & \\
\hline $2^{-+}$ & $\eta_{2}(1645)$ & $1617 \pm 5$ & & $\bar{q} q_{020}^{\eta}$ & 1.58 & & & \\
\hline $2^{++}$ & $f_{2}(1270)$ & 1276 & & $\bar{q} q_{011}$ & 1.29 & & & $a_{2}(1320)$ \\
\hline $2^{++}$ & $f_{2}^{\prime}(1525)$ & $1525 \pm 5$ & d & $\bar{s} s_{011}$ & 1.49 & & & \\
\hline $2^{++}$ & $f_{2}(1950)$ & $1944 \pm 12$ & & $\bar{s} s_{111}$ & 1.82 & & & \\
\hline $2^{++}$ & $f_{2}(2010)$ & $2011 \pm 70$ & ss & $\bar{s} s_{211}$ & 2.10 & $(\overline{s s} s s)_{002}$ & 2.13 & \\
\hline $2^{++}$ & $f_{2}(2300)$ & $2297 \pm 28$ & ss & $\bar{s} s_{311}$ & 2.35 & & & \\
\hline $2^{++}$ & $f_{2}(2340)$ & $2345 \pm 50$ & Ss & & & $(\overline{S S} S S)_{102}$ & 2.37 & \\
\hline $3^{--}$ & $\omega_{3}(1670)$ & $1667 \pm 4$ & & $\bar{q} q_{021}$ & 1.66 & & & $\rho_{3}(1690)$ \\
\hline $3^{--}$ & $\phi_{3}(1850)$ & $1854 \pm 7$ & & $\bar{s} s_{021}$ & 1.82 & & & \\
\hline $4^{++}$ & $f_{4}(2050)$ & $2018 \pm 11$ & & $\bar{q} q_{031}$ & 1.96 & & & $a_{4}(2040)$ \\
\hline
\end{tabular}

$$
\begin{aligned}
M_{\eta^{\prime}}^{2}= & \Delta_{\eta}^{2} \lambda+\Delta M^{2}\left[m_{s}, m_{s}\right]=M_{\eta}^{2}-\Delta^{2}\left[m_{q}, m_{q}\right] \\
& +\Delta^{2}\left[m_{s}, m_{s}\right] .
\end{aligned}
$$

Using the results of Table I, Eq. (11) leads to $M_{\eta^{\prime}}=$ $0.937 \mathrm{GeV}$, in good agreement with the experimental value $M_{\eta^{\prime}}=0.958 \pm 0.06 \mathrm{GeV}$ [27].

In Fig. 2 the theoretical trajectories for the $\eta, \eta^{\prime}$, and $h$ mesons and their radial and orbital excitations are shown. Since not very many $\eta$ mesons, and even fewer $h$ mesons, have been confirmed experimentally, we have also considered unconfirmed states listed in [27]. As can be seen from Fig. 2, the agreement between theory and experiment is for the ground states indeed satisfactory, particularly the $\eta-\eta^{\prime}$ mass difference is explained. As in other quark-spin zero cases the agreement between the simple theory and experiment is not so good for radial excitations. Presumably mixing plays some role there. For a more detailed discussion, see the next subsection.

\section{B. General comparison of isoscalar bosons}

In Table III we show all of the confirmed isoscalar bosons. In the first four columns we show the experimental results; the letter $d$ in the column "Decay" indicates that decay channels with open or hidden strangeness are dominant, and ss indicates that there are decay channels with fourfold hidden strangeness, such as the $\phi \phi$, for the $\eta^{\prime}$ 
TABLE IV. A compilation of the tetraquark states and their partners. States omitted from the summary table of PDG are marked by a question mark ? The indices of the quark content denote $n, L, \mathcal{S}$, the radial excitation, LF momentum, and the quark or diquark spin. The differences of the theoretical masses, $M_{\text {theo }}$ shows the amount of SUSY breaking due to the additional mass terms. Assignments with remark $C$ do not explain the occurrence of isovector states with the same residual quantum numbers and similar masses.

\begin{tabular}{|c|c|c|c|c|c|c|c|c|c|}
\hline \multirow[b]{2}{*}{ Name } & \multicolumn{2}{|l|}{ Tetraquark } & \multicolumn{3}{|c|}{ Baryon } & \multicolumn{3}{|c|}{ Meson } & \multirow[t]{2}{*}{ Remark } \\
\hline & Quark content & $M_{\text {theo }}$ & Name & Quark content & $M_{\text {theo }}$ & Name & Quark content & $M_{\text {theo }}$ & \\
\hline$\overline{f_{0}(980)}$ & $(\overline{q q} q q)_{000}$ & 1.10 & $N(940)$ & $(q q q)_{000}$ & 1.07 & $h_{1}(1170)$ & $\bar{q} q_{010}^{\eta}$ & 1.18 & $\mathrm{C}$ \\
\hline$\omega(1420)$ & $(\overline{q q} q q)_{010}$ & 1.52 & $N^{\frac{1}{2}}-(1535)$ & $(q q q)_{010}$ & 1.50 & $\eta_{2}(1645)$ & $\bar{q} q_{020}^{\eta}$ & 1.58 & $\mathrm{C}$ \\
\hline$f_{0}(1500)$ & $(\overline{q q} q q)_{100}$ & 1.52 & $N^{\frac{1}{2}+}(1440)$ & $(q q q)_{100}$ & 1.50 & $h_{1}(1595)^{?}$ & $\bar{q} q_{110}^{\eta}$ & 1.58 & \\
\hline$f_{1}(1420)$ & $(\overline{s q} s q)_{001}$ & 1.6 & $\Xi^{\frac{3}{2}}+(1530)$ & $(s s q)_{001}$ & 1.50 & $f_{2}^{\prime}(1525)$ & $\bar{s} s_{011}$ & 1.49 & \\
\hline$\phi(1680)$ & $(\overline{s q} s q)_{010}$ & 1.76 & $\Xi^{*}(1690)^{?}$ & $(s s q)_{010}$ & 1.67 & $\eta_{2}(1870)^{?}$ & $\bar{s} s_{020}^{\eta}$ & 1.75 & \\
\hline
\end{tabular}

decay (see the discussion below). In the four columns listed under "Theory" we show below $M_{n L, J-L}$ and $T_{n L, J-L}$ the quark content, the radial excitation number $n$, the light front angular momentum $L$, and the difference $J-L$, as indices. For the $\eta$-like mesons with $J^{P C}=0^{-+}$or $1^{+-}$we denote by an upper index $\eta$ the fact that the shift given by $\Delta_{\eta}^{2} \lambda$ (see Sec. II) has to be performed; the theoretical masses are calculated according to [(6), (7)] and [(9), (10)], respectively.

We will leave out from this comparison the extremely broad $f_{0}(500)$ which will be discussed later. The overall fit from theory to experiment is satisfactory-the standard deviation (SD) between theory and experiment is $\mathrm{SD}=93 \mathrm{MeV}$, well inside the model uncertainty of $\approx 100 \mathrm{MeV}$, as expected from the $N_{C} \rightarrow \infty$ expansion [28]. The discrepancy between theory and experiment is no more than 3 standard deviations for any of the considered 27 states. Therefore we accept only as probable a tetraquark assignment for states where the difference between the experimental masses and LF holographic predictions is less than $3 \mathrm{SD} \approx 280 \mathrm{MeV}$.

We now start a detailed discussion of the states:

(a) $\eta$ and $h$ mesons, $J^{P C}=0^{-+}, 1^{+-}, 2^{-+}$. In our approach the $\eta$ is predominantly $\bar{q} q$ and the $\eta^{\prime}$ is predominantly $\bar{s} s$. Since the $\eta$ is slightly below the $4 \pi$ threshold and the $\eta^{\prime}$ below the $K \bar{K}$ threshold, we cannot test this assignment by the decays, but the width of the $\eta^{\prime}(958)$ meson is very small $(196 \mathrm{keV})$. The dominant decay $\eta \pi \pi$ is not forbidden by any selection rule or spin and statistics. This is an additional argument that $\eta$ and $\eta^{\prime}$ have a different quark content, with the $\eta^{\prime}$ having an important hidden strangeness. As mentioned in Sec. II A, the agreement between theory and experiment is satisfactory. Presumably, the $\eta(1295), \eta(1405)$ and the $\eta(1475)$ are mixed states of the $\eta(2 S), \eta^{\prime}(2 S)$ and $\eta(3 S)$.

(b) $f_{0}$ states. The assignment of $f_{0}(500)$ and $f_{0}(980)$ as members of a nonet of tetraquarks, with and without hidden strangeness, appears very plausible [6,29-33]; however, quantitative predictions from LFHQCD do not add support to this assignment: The lightest tetraquark consisting of nonstrange quarks, $(\overline{q q} q q)_{000}$, has a mass of $1.10 \mathrm{GeV}$, compatible with the $f_{0}(980)$, but the lightest tetraquark with hidden strangeness, $(\overline{s q} s q)_{000}$, has a mass of $1.42 \mathrm{GeV}$. The conventional meson in a $q \bar{q}\left({ }^{3} P_{0}\right)$ state, $\bar{q} q_{01-1}$, has in LFHQCD the mass $0.75 \mathrm{GeV}$ which is also in this mass range. Unfortunately, the quantitative predictions from LFHQCD do not contribute to the solution of this interesting situation.

The other $f_{0}$ states fit very well into the LFHQCD theoretical scheme. The $f_{0}(1500)$ is a very good candidate for a radially excited tetraquark; its mass fits nicely, and its baryonic partner is the Roper resonance $N(1440)$ (see Table IV).

(c) $f_{1}$ states. The situation is similar as for the $f_{0}(980)$. The $f_{1}(1282)$ is heavier than the ${ }^{1} P_{1}$ ground state $(1.06 \mathrm{GeV})$ and lighter than its first radial excitation $(1.49 \mathrm{GeV})$, there is also no tetraquark with fitting mass, and the occurrence of the near degenerate isospin partner $a_{1}(1260)$ makes an interpretation as a meson state plausible anyhow. An interpretation of the $f_{1}(1282)$ and $a_{1}(1260)$ as tetraquarks with $\mathcal{S}=1$ and hidden strange $(\overline{s q} s q)_{001}[6]$ is not supported by the quantitative LFHQCD analysis, the discrepancy between the theoretical mass value for this assignment and experiment is $315 \mathrm{MeV} \sim 3.6 \mathrm{SD}$.

(d) $f_{2}$ states. Our quark assignment in Table III for the $f_{2}(1270)$ and the $f_{2}^{\prime}(1525)$ is compatible with a recent lattice computation above threshold, where the lighter state couples predominantly to $\pi \pi$ and the heavier to $K \bar{K}$ [34]. On the other hand, the profusion of $f_{2}$ states makes the occurrence of exotic states very plausible. Furthermore the occurrence of two $\phi$ mesons in the decay channels of the heavier $f_{2}$ mesons strongly suggests the admixture of a tetraquark consisting of two strange and two antistrange quarks. As mentioned in Sec. II A, a diquark cluster with strangeness \pm 2 must have spin 1 , so it is plausible that these states contribute to mesons with $J=2$. Extending (7) also to 
diquarks with spin 2, we obtain for the lightest tetraquarks with quark content $(\overline{s S} s s)_{002}$ the mass $M_{T}=2.13 \mathrm{GeV}$, and for the $(\overline{s s} s)_{102}$ configuration $M_{T}=2.37 \mathrm{GeV}$; they are just in the mass range of those states which decay into two $\phi$ mesons. We therefore propose that the $f_{2}(2010), f_{2}(2300)$, and $f_{2}(2340)$ are mixtures of normal meson states and tetraquark states, as indicated in Table III. These tetraquarks with aligned spin have positive parity and charge parity $P=C=1$.

In Table IV we have listed those tetraquark states which fit quantitatively into the scheme of supersymmetric LFHQCD. The masses of the first two, the $f_{0}(980)$ and the $\omega(1420)$, fit reasonably well into the LFHQCD scheme, although the isospin degeneracy with the $a_{0}(980)$ and the $\rho(1440)$ cannot be explained by this assignment. The remaining four states are, however, very probably tetraquark states. Note that the additional mass correction term leads to a breaking of the supersymmetry, as can be seen from the different theoretical masses inside one supermultiplet.

\section{SUMMARY AND DISCUSSION}

As has been shown in Sec. II A, the addition of a constant term to the $I=0, \mathcal{S}=0$ bosonic sector of the supersymmetric light front holographic Hamiltonian provides an explanation of the entire $\eta-h$ spectrum. This additional term, which breaks the supersymmetry of the spectra, plays the role of a direct chiral $U(1)$ breaking term in effective chiral representations of standard QCD. In fact, at the classical level, the QCD Lagrangian with massless up and down quarks is invariant under $U_{L}(2) \otimes U_{R}(2)$ transformations. In conventional effective chiral theory, $S U_{L} \otimes$ $S U_{R}(2)$ is broken spontaneously and leads to an isovector of Goldstone particles which are identified with the $\pi$ mesons. Since the isoscalar pseudoscalar meson, the $\eta$ meson, is considerably heavier than $\sqrt{3} M_{\pi} \approx 237 \mathrm{MeV}$ [35], there is apparently no Goldstone boson of the remaining chiral $U(1)$ symmetry and it is most probably broken directly, for instance by instanton solutions or other nonperturbative effects, which modify the effective QCD Hamiltonian [18-26].

In supersymmetric LFHQCD, the implementation of the superconformal algebra is the origin of the vanishing mass of mesons with $L=0, \mathcal{S}=0$, since it predicts a constant term $-2 \lambda$ in the LF potential which exactly cancels the LF kinetic energy. Since the lowest meson state has no supersymmetric partners [2], it plays the role of a zeroenergy nondegenerate ground state. This occurrence of massless mesons is conventionally associated with the spontaneous breaking of the chiral $S U(2)$ symmetry of QCD based on effective meson fields.

But if the chiral $U(1)$ symmetry is broken directly, the supersymmetry of LFHQCD also needs a hard breaking. Such a symmertry breaking term can most easily be incorporated into light front holographic QCD by adding to the LF potential $U_{\mathrm{AdS}}(\zeta)$ [see (A1)] for the bosonic channels the constant term $\lambda \Delta_{\eta}^{2} \delta_{\mathcal{S} 0} \delta_{I 0} \approx \lambda \delta_{\mathcal{S} 0} \delta_{I 0}$ (8). It is remarkable that this simple modification of the potential, which is determined by the eta mass [see (8)], can explain the full $\eta$ and $h$ meson spectrum, including in particular the $\eta^{\prime}-\eta$ mass difference, as shown in Fig. 2. It should be noted that also in more standard analytical approaches to the $U(1)$ problem [36] at least one additional parameter is necessary, be it an instanton cutoff $[19,37]$ or mixing angles [38]. In the channels with quark spin $\mathcal{S}=1$ no symmetrybreaking term is present and no modification of supersymmetric LFHQCD is necessary.

We have also considered states with $J \neq L+\mathcal{S}$, which are not members of supermultiplets; their masses are predicted by LFHQCD, given the modification of the AdS action which is determined by the implementation of the superconformal algebra. The theoretical mass predictions are collected in Fig. 2 and Table III.

The quantitative discussion of tetraquarks in this investigation is complementary to that of Ref. [6], where a general qualitative overview of possible supermultiplets in all channels was given. In this paper we have concentrated on the possible tetraquark states, the mass of which agrees within the model accuracy of $\approx 100 \mathrm{MeV}$ with the theoretical predictions. Candidates fulfilling these criteria are collected in Table IV, together with their partners. The most convincing candidate is the $f_{0}(1500)$. The $f_{0}(1500)$ has also been considered as a candidate for a glueball (see, e.g., $[39,40])$; it should be noted, however, that in LFHQCD there is no sign of valence gluons, and the identification of the $f_{0}(1500)$ as a tetraquark is quantitatively very convincing.

In this article we have concentrated on the new insights that LFHQCD brings to the symmetry breaking mechanisms of standard QCD. We have also emphasized the remarkable numerical coincidence of the magnitude of the "symmetry breaking" term $\lambda \Delta \eta^{2}$ with the value of the confinement scale $\lambda$ of LFHQCD. In fact, the results described in this article provide a new correspondence between the chiral symmetries of standard QCD and LFHQCD: In the conventional approach, the occurrence of a massless pseudoscalar particle in the massless quark limit is assumed to be a consequence of the Goldstone theorem, whereas in supersymmetric LFHQCD, the massless of the composite $q \bar{q}$ pion bound state is an explicit feature of the lowest eigenvalue of the LF Hamiltonian. Superconformal LFHQCD thus leads to a novel alternative mechanism for the origin of massless composite pseudoscalar mesons.

The explicit breaking of the $U(1)$ classical symmetry, whatever its reason in standard QCD, can be incorporated into LFHQCD by adding a constant to the superconformal potential. The confining term $\lambda \zeta^{2}$, which is a consequence of the conformal algebra alone [41], is not affected. The remarkable fact that the symmetry breaking term $\lambda \Delta_{\eta}^{2}$ is 
equal within errors to the only scale available in LFHQCD in the chiral limit, Eq. (8), indicates a deep connection of universal mass scales in QCD.

\section{ACKNOWLEDGMENTS}

One of us (H. G. D.) thanks the Chinese Academy of Sciences and especially Professor Pengming Zhang for the warm hospitality extended to him at the Institute of Modern Physics, Lanzhou (Gansu). This work is supported in part by the Department of Energy, Contract No. DEAC02-76SF00515 and by the National Natural Science Foundation of China (Grants No. 11575254 and No. 11805242).

\section{APPENDIX: SUMMARY OF LFHQCD RESULTS}

In this appendix we summarize the relevant equations from LFHQCD which underlie the theoretical foundations of this paper. The equations of motion for a meson with arbitrary spin $J$, represented by a fully symmetric tensor field of rank- $J$ in five-dimensional AdS space, follows from an $\mathrm{AdS}_{5}$ action with the soft-wall dilaton term $e^{\varphi(z)}$ [17]:

$$
\left(-\frac{d^{2}}{d z^{2}}+\frac{4 L_{\mathrm{AdS}}^{2}-1}{4 z^{2}}+U_{\mathrm{AdS}}(z)\right) \Phi_{J}(z, q)=q^{2} \Phi_{J}(z, q) .
$$

Here $z$ is the fifth coordinate of $\mathrm{AdS}_{5}$, the holographic variable in the five-dimensional space, and $q^{2}$ is the momentum in the four-dimensional (physical) spacetime. The potential $U_{\mathrm{AdS}}(z)$ depends on the dilaton profile $\varphi(z)$ :

$$
U_{\mathrm{AdS}}(z)=\frac{1}{2} \varphi^{\prime \prime}(z)+\frac{1}{4}\left(\varphi^{\prime}(z)\right)^{2}+\frac{2 J-3}{2 \zeta} \varphi^{\prime}(z) .
$$

The quantity $L_{\text {AdS }}^{2}$ is determined by $J$ and the dimensionless product of the AdS-mass $\mu$ with the space curvature $R: L_{\text {AdS }}^{2}=(\mu R)^{2}+(2-J)^{2}$.

The form of (A1) is that of a bound-state equation for a hadron consisting of two massless constituents in light front quantization. The holographic variable $z$ is identified with the boost-invariant LF variable $\zeta=\sqrt{x(1-x)} b_{\perp}$, where $x$ is the longitudinal momentum fraction of one of the quark constituents and $b_{\perp}$ is the transverse separation of the constituents (quarks or quark clusters) in the transverse plane. The LF angular momentum $L$ is identified with the quantity $L_{\mathrm{AdS}}$, and the light front potential $U(\zeta)$ is therefore determined by (A2).

If one implements superconformal algebra [1,2] by requiring the LF Hamiltonian to be a superposition of the generators of the superconformal algebra following $[42,43]$, the form of the LF potential is completely fixed to

$$
U(\zeta)=\lambda^{2} \zeta^{2}+2 \lambda(L-1)
$$

which leads to eigenvalues

$$
M^{2}=|\lambda|(4 n+2 L+2)+2 \lambda(L-1) .
$$

The potential derived from the implementation of the superconformal algebra is only compatible with the holographic approach if the dilaton profile $\phi(z)=\lambda z^{2}$ and holds for mesons with $J=L$. It is remarkable that this choice of maximal symmetry breaking is the one which had been chosen before in the soft wall model [44] for purely phenomenological reasons, namely to generate linear Regge trajectories for mesons. A zero mass state occurs only if the sign of $\lambda$ is positive,

$$
M^{2}=4 \lambda(n+L),
$$

and therefore the lowest meson state has no baryon partner $[2,45]$. One sees immediately that in order to get the harmonic part of the potential from (A2), one has to choose the $\varphi(\zeta)=\lambda \zeta^{2}$, and one obtains in this case

$$
U_{\mathrm{AdS}}(\zeta)=\lambda^{2} \zeta^{2}+2 \lambda(J-1) .
$$

We can thus extend the superconformal approach to mesons with quark spin $\mathcal{S}=1$ and $J=L+1$ by adding the term $2 \lambda \mathcal{S}$ to (A3), to recover the result (A6) for mesons with both $\mathcal{S}=0$ and $\mathcal{S}=1, J=L+\mathcal{S}$. Therefore our final result for the LF potential, valid for mesons with $J=L+\mathcal{S}$, is

$$
U(\zeta)=\lambda^{2} \zeta^{2}+2 \lambda(L+\mathcal{S}-1)
$$

and the resulting meson spectrum is the one given by (1).

The implementation of the superconformal algebra implies [42,43] that besides the Hamiltonian for the bosonic wave function, there is also one for a fermionic one, which describes the supersymmetric fermion of the boson described by (A3). Its potential is

$$
U(\zeta)=\lambda^{2} \zeta^{2}+2 \lambda(L+1)
$$

and leads to the eigenvalues $[1,2]$

$$
M^{2}=4 \lambda(n+L+1) .
$$

Consequently a $q \bar{q}$ meson with angular momentum $L_{M}$ has the same mass as a baryon with angular momentum $L_{B}=$ $L_{M}+1$ between its quark and diquark cluster components. This relation has been tested and is very well satisfied for many spectra of light and even heavy hadrons $[2,3,5,6]$. The superconformal baryon potential (A8) can also be obtained from an AdS action for fermion fields if a Yukawa-like term $\bar{\Psi} z \Psi$ is added to the Lagrangian. This modification had been introduced earlier for purely phenomenological reasons $[46,47]$. The Hamiltonian (A8) in this case applies to the positively aligned chirality component $\psi^{+}$of the baryon. 
There is also the negative chirality component $\psi^{-}$of the baryon. The corresponding LF potentials are [1]

$$
U_{+}(\zeta)=\lambda^{2} \zeta^{2}+2 \lambda(L+1)
$$

namely Eq. (A8) for the positive component, and

$$
U_{-}(\zeta)=\lambda^{2} \zeta^{2}+2 \lambda L
$$

for the negative component. The LF potential, together with the term $2 \lambda \mathcal{S}$ introduced above, leads to the baryon spectrum given in (2).
Finally, there is also a bosonic superpartner of the negative chirality component $\psi^{-}$of the baryon, which is interpreted as a tetraquark $[3,6,16]$. Its mass spectrum is given in (3). The meson, positive and negative chiral baryon states $\psi^{ \pm}$, and the tetraquark form a 4-plet supermultiplet.

We can generalize the results (1), (2), and (3) obtained in supersymmetric LFHQCD by going back to normal LFHQCD. Once the modification is fixed, we can use the specific AdS result (A6) and obtain for the meson spectrum Eq. (6).
[1] G. F. de Téramond, H. G. Dosch, and S. J. Brodsky, Baryon spectrum from superconformal quantum mechanics and its light-front holographic embedding, Phys. Rev. D 91, 045040 (2015).

[2] H. G. Dosch, G. F. de Téramond, and S. J. Brodsky, Superconformal baryon-meson symmetry and light-front holographic QCD, Phys. Rev. D 91, 085016 (2015).

[3] S. J. Brodsky, G. F. de Téramond, H. G. Dosch, and C. Lorcé, Universal effective hadron dynamics from superconformal algebra, Phys. Lett. B 759, 171 (2016).

[4] H. G. Dosch, G. F. de Téramond, and S. J. Brodsky, Supersymmetry across the light and heavy-light hadronic spectrum, Phys. Rev. D 92, 074010 (2015).

[5] H. G. Dosch, G. F. de Téramond, and S. J. Brodsky, Supersymmetry across the light and heavy-light hadronic spectrum II, Phys. Rev. D 95, 034016 (2017).

[6] M. Nielsen and S. J. Brodsky, Hadronic superpartners from a superconformal and supersymmetric algebra, Phys. Rev. D 97, 114001 (2018).

[7] M. Nielsen, S. J. Brodsky, G. F. de Téramond, H. G. Dosch, F. S. Navarra, and L. Zou, Supersymmetry in the doubleheavy hadronic spectrum, Phys. Rev. D 98, 034002 (2018).

[8] S. J. Brodsky and G. F. de Téramond, Light-front hadron dynamics and AdS/CFT correspondence, Phys. Lett. B 582, 211 (2004).

[9] G. F. de Téramond and S. J. Brodsky, Light-Front Holography: A First Approximation to QCD, Phys. Rev. Lett. 102, 081601 (2009).

[10] S. J. Brodsky, G. F. de Téramond, H. G. Dosch, and J. Erlich, Light-front holographic QCD and emerging confinement, Phys. Rep. 584, 1 (2015).

[11] G. F. de Téramond, T. Liu, R. S. Sufian, H. G. Dosch, S. J. Brodsky, and A. Deur, Universality of generalized parton distributions in light-front holographic QCD, Phys. Rev. Lett. 120, 182001 (2018).

[12] R. S. Sufian, T. Liu, G. F. de Téramond, H. G. Dosch, S. J. Brodsky, A. Deur, M. T. Islam, and B. Q. Ma, Nonperturbative strange-quark sea from lattice QCD, light-front holography, and meson-baryon fluctuation models, Phys. Rev. D 98, 114004 (2018).
[13] J. M. Maldacena, The large-N limit of superconformal field theories and supergravity, Int. J. Theor. Phys. 38, 1113 (1999).

[14] S. J. Brodsky and G. F. de Téramond, Hadronic Spectra and Light-Front Wave Functions in Holographic QCD, Phys. Rev. Lett. 96, 201601 (2006); Light-front dynamics and AdS/QCD correspondence: The pion form factor in the space- and timelike regions, Phys. Rev. D 77, 056007 (2008).

[15] S. J. Brodsky and G. F. de Téramond, Light-front dynamics and AdS/QCD correspondence: Gravitational form factors of composite hadrons, Phys. Rev. D 78, 025032 (2008).

[16] L. Zou and H. G. Dosch, A very practical guide to light front holographic QCD, arXiv:1801.00607.

[17] G. F. de Téramond, H. G. Dosch, and S. J. Brodsky, Kinematical and dynamical aspects of higher-spin bound-state equations in holographic QCD, Phys. Rev. D 87, 075005 (2013).

[18] J. B. Kogut and L. Susskind, Quark confinement and the puzzle of the ninth axial-vector current, Phys. Rev. D 10, 3468 (1974).

[19] G. 't Hooft, Symmetry Breaking through Bell-Jackiw Anomalies, Phys. Rev. Lett. 37, 8 (1976); How instantons solve the U(1) problem, Phys. Rep. 142, 357 (1986).

[20] E. Witten, Current algebra theorems for the U(1) Goldstone boson, Nucl. Phys. B156, 269 (1979).

[21] G. Veneziano, U(1) without instantons, Nucl. Phys. B159, 213 (1979).

[22] G. Veneziano, Goldstone mechanism from gluon dynamics, Phys. Lett. 95B, 90 (1980).

[23] P. Di Vecchia and G. Veneziano, Chiral dynamics in the large $N$ limit, Nucl. Phys. B171, 253 (1980).

[24] M. Engelhardt, Center vortex model for the infrared sector of Yang-Mills theory: Topological susceptibility, Nucl. Phys. B585, 614 (2000).

[25] R. Alkofer, C. S. Fischer, and R. Williams, $U(1)_{A}$ anomaly and $\eta^{\prime}$-mass from an infrared singular quark-gluon vertex, Eur. Phys. J. A 38, 53 (2008).

[26] A. L. Kataev, N. V. Krasnikov, and A. A. Pivovarov, The connection between the scales of the gluon and quark worlds in perturbative QCD, Phys. Lett. 107B, 115 (1981); Two-loop calculations for the propagators of gluonic currents, Nucl. 
Phys. B198, 508 (1982); Erratum, Nucl. Phys. B490, 505(E) (1997).

[27] M. Tanabashi et al. (Particle Data Group), Review of particle physics, Phys. Rev. D 98, 030001 (2018).

[28] G. 't Hooft, A planar diagram theory for strong interactions, Nucl. Phys. B72, 461 (1974).

[29] G. 't Hooft, G. Isidori, L. Maiani, A. D. Polosa, and V. Riquer, A theory of scalar mesons, Phys. Lett. B 662, 424 (2008).

[30] D. Black, A. H. Fariborz, and J. Schechter, Mechanism for a next-to-lowest lying scalar meson nonet, Phys. Rev. D 61 , 074001 (2000).

[31] R. L. Jaffe and F. Wilczek, Diquarks and Exotic Spectroscopy, Phys. Rev. Lett. 91, 232003 (2003).

[32] R. L. Jaffe, Multiquark hadrons. 1. Phenomenology of $Q^{2} \bar{Q}^{2}$ mesons, Phys. Rev. D 15, 267 (1977).

[33] L. Maiani, F. Piccinini, A. D. Polosa, and V. Riquer, A New Look at Scalar Mesons, Phys. Rev. Lett. 93, 212002 (2004).

[34] R. A. Briceno, J. J. Dudek, R. G. Edwards, and D. J. Wilson, Isoscalar $\pi \pi, K \bar{K}, \eta \eta$ scattering and the $\sigma, f_{0}, f_{2}$ mesons from QCD, Phys. Rev. D 97, 054513 (2018).

[35] S. Weinberg, The U(1) problem, Phys. Rev. D 11, 3583 (1975).

[36] G. A. Christos, Chiral symmetry and the U(1) problem, Phys. Rep. 116, 251 (1984).
[37] A. A. Belavin, A. M. Polyakov, A. S. Schwartz, and Y. S. Tyupkin, Pseudoparticle solutions of the Yang-Mills equations, Phys. Lett. 59B, 85 (1975).

[38] R. Escribano and J. M. Frère, Study of the $\eta-\eta^{\prime}$ system in the two mixing angle scheme, J. High Energy Phys. 06 (2005) 029.

[39] C. Amsler and F. E. Close, Is $f_{0}(1500)$ a scalar glueball?, Phys. Rev. D 53, 295 (1996).

[40] F. E. Close and A. Kirk, The mixing of the $f_{0}(1370)$, $f_{0}(1500)$ and $f_{0}(1710)$ and the search for the scalar glueball, Phys. Lett. B 483, 345 (2000).

[41] S. J. Brodsky, G. F. de Téramond, and H. G. Dosch, Threefold complementary approach to holographic QCD, Phys. Lett. B 729, 3 (2014).

[42] V. de Alfaro, S. Fubini, and G. Furlan, Conformal invariance in quantum mechanics, Nuovo Cimento A 34, 569 (1976).

[43] S. Fubini and E. Rabinovici, Superconformal quantum mechanics, Nucl. Phys. B245, 17 (1984).

[44] A. Karch, E. Katz, D. T. Son, and M. A. Stephanov, Linear confinement and AdS/QCD, Phys. Rev. D 74, 015005 (2006).

[45] E. Witten, Dynamical breaking of supersymmetry, Nucl. Phys. B188, 513 (1981).

[46] I. Kirsch, Spectroscopy of fermionic operators in AdS/CFT, J. High Energy Phys. 09 (2006) 052.

[47] Z. Abidin and C. E. Carlson, Nucleon electromagnetic and gravitational form factors from holography, Phys. Rev. D 79, 115003 (2009). 